\title{
WHO 2006 child growth standards: implications for the prevalence of stunting and underweight-for-age in a birth cohort of Gabonese children in comparison to the Centers for Disease Control and Prevention 2000 growth charts and the National Center for Health Statistics 1978 growth references
}

Norbert G Schwarz 1,2, Martin P Grobusch 1,3, Marie-Luise Decker ${ }^{1,2}$, Julia Goesch ${ }^{1,2}$, Marc Poetschke ${ }^{1,2}$, Sunny Oyakhirome ${ }^{1}$, Davy Kombila', Julien Fortin ${ }^{1}$, Bertrand Lell ${ }^{1,2}$, Saadou Issifou', Peter $G$ Kremsner ${ }^{1,2}$ and Kerstin Klipstein-Grobusch ${ }^{4, *}$ ${ }^{1}$ Medical Research Unit, Albert Schweitzer Hospital, Lambaréné, Gabon: ${ }^{2}$ Department of Parasitology, Institute of Tropical Medicine, University of Tübingen, Germany: ${ }^{3}$ Infectious Diseases Unit, CMID and NHLS, Faculty of Health Sciences, University of the Witwatersrand, Johannesburg, South Africa: ${ }^{4}$ School of Public Health, Faculty of Health Sciences, University of the Witwatersrand, York Road 7, Parktown, Johannesburg 2193, South Africa

Submitted 30 March 2007: Accepted 24 October 2007: First published online 2 January 2008

\begin{abstract}
Objectives: To assess the proportion of children being stunted and underweightfor-age at 3, 9 and 15 months in Lambaréné, Gabon, using the WHO child growth standards released in 2006 as compared with the Centers for Disease Control and Prevention (CDC) 2000 and the National Center for Health Statistics (NCHS) 1978 child growth charts/references.

Design and setting: Prospective birth cohort in Lambaréné, Gabon.

Subjects: Two hundred and eighty-nine children from birth to 15 months of age. Methods: Weight and length were recorded at 3, 9 and 15 months. Corresponding $Z$ scores for stunting and underweight-for-age were calculated for the three different standards/references. Children with a height-for-age or weight-for-age below -2 SD of the corresponding reference median $(Z$ score $\leq-2)$ were classified as stunted or underweight-for-age, respectively.

Results: With the new WHO 2006 standards a higher proportion $(4 \cdot 0 \%)$ of 3 -month-old infants were underweight compared with the CDC $(1 \cdot 0 \%)$ or the NCHS $(0 \cdot 7 \%)$ child growth charts/references. In contrast to the NCHS references or the CDC charts, this proportion did not increase from 3 to 9 months or from 9 to 15 months. The proportion of children being stunted was highest (above 20\%) with the WHO 2006 standards at all three ages. Again, in contrast to the old standards, this proportion did not increase from 3 to 9 months or from 9 to 15 months.

Conclusions: The present results show considerably different growth faltering patterns for Gabonese children depending on the growth charts used to assess the prevalence of stunting and underweight. Shifting to the new WHO child growth standards may have important implications for child health programmes.
\end{abstract}

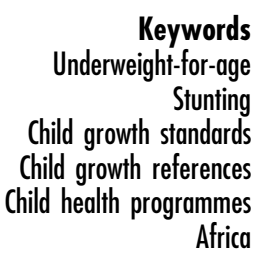

Africa
In April 2006 the WHO released new child growth standards for infants and children up to the age of 5 years to replace the National Center for Health Statistics (NCHS) 1978 growth references ${ }^{(1)}$ and the Centers for Disease Control and Prevention (CDC) 2000 growth charts ${ }^{(2)}$ so far commonly used to assess the nutritional status of children in nutritional surveys all over the world ${ }^{(3,4)}$. They are based on the WHO Multicentre Growth Reference Study ${ }^{(5-8)}$, a population-based study conducted between 1997 and 2003 in about 8500 children from six different countries (Brazil, Ghana, India, Norway, Oman and the USA). Children were followed up longitudinally from 0 to 24 months with measurements at birth, weeks 1 to 4 and 6 , then monthly from month 2 to month 12 , and bimonthly in the second year. The data from 18 to 71 months were collected as cross-sectional data. The aim was to generate growth curves that represent the best description of physiological growth from birth to 5 years of age in breast-fed infants and establish the breast-fed infant as the normative model for growth and development. Consequently, only 
those children who were exclusively breast-fed for 4 months, acquainted with solid foods at 6 months and breast-fed throughout the whole first year of life were included (http://www.who.int/childgrowth/en/).

The NCHS 1978 growth references were the normalized version produced by the CDC based on the growth charts developed a year before by the NCHS. They were subsequently recommended by the WHO for international application and therefore used in nutritional surveys all over the world ${ }^{(9,10)}$. This version made it possible to express height-for-age and weight-for-age in terms of standard deviations from the median or $Z$ score $^{(1,11,12)}$. The NCHS standards were based on cross-sectional data from three National Health and Nutrition Examination Surveys and on the data from the Fels Study, a longitudinal study carried out from 1929 to 1975 in southwestern Ohio by the Fels Research Institute (Yellow Springs, OH, USA). The Fels data were used to produce the charts for children less than 36 months of age. They came from a single regional population of mainly formulafed, white, middle-class infants and were therefore criticized for not being representative ${ }^{(13)}$. Measurements were taken at birth, 1 month and 3 months, and then at 3-month intervals from 3 to 12 months and at 6-month intervals from 12 to 36 months: intervals possibly not close enough in periods of rapid change. Further concerns were about the right skewed (obesity) distribution.

The CDC 2000 growth reference charts were based on data from five cross-sectional national health surveys from the USA ${ }^{(2)}$ supplemented by birth weight data from US birth certificates and birth length data from the Missouri and Wisconsin Vital Statistics. Babies with a birth weight under $1500 \mathrm{~g}$ were excluded. Again the main concerns about these standards centred on the infant age group: birth weight and birth length were from different data sources, and there were no data available between birth and 2 months of age. The sample size for children under 6 months was small and both the NCHS references and the CDC growth charts were based on relatively few infants who were breast-fed for longer than a few months ${ }^{(14,15)}$.

Both the NCHS and the CDC child growth references/ charts reflected how growth was in the populations the data were taken from, whereas the WHO 2006 child growth standards are set out to reflect how growth should be.

Based on a birth cohort of 289 children from Lambaréné, Gabon whose weight and length were measured at the age of 3,9 and 15 months, the present study compares the proportion of children being underweight-for-age and the proportion of children being stunted at the age of 3,9 and 15 months according to the WHO 2006, the CDC and the NCHS growth references.

\section{Methods}

At the age of 3,9 and 15 months we recorded the weight and height of children who were followed up in a longitudinal study to assess the efficacy (regarding the outcomes malaria and anaemia) and safety of intermittent presumptive treatment in infants through sulphadoxine pyrimethamine administered at the age of 3,9 and 15 months that had started in December 2002 ${ }^{(16-18)}$. Written consent was obtained from all primary caregivers of all study children enrolled. Ethical clearance was granted from the Ethics Committees of the International Foundation of the Albert Schweitzer Hospital in Lambaréné, Gabon, and the University of Tübingen, Germany. Four hundred and one children were included in a substudy on socio-economic risk factors for stunting and underweight-for-age which was carried out between December 2004 and July 2005. The data of 289 children with complete measurements of weight and height at birth and at the age of 3,9 and 15 months were included in the analysis. At 3 months of age babies were weighed in a lying position, at 9 and 15 months of age sitting on a balance. Weight was measured with the same mechanical baby balance that was on a fixed horizontal surface throughout the whole study. Calibration of the balance was checked before every session. Length measurements were carried out with a cloth tape on the infant in a lying position, measuring from head to heel, at the age of 3 and 9 months, and with the infant in standing position without shoes at the age of 15 months ${ }^{(19)}$. Anthropometric measurements were conducted by trained health professionals following standardized procedures. Based on this birth cohort we

Table 1 Description of the study population of children from Lambaréné, Gabon

\begin{tabular}{|c|c|c|c|c|c|c|}
\hline & \multicolumn{2}{|c|}{ Pooled (n 289) } & \multicolumn{2}{|c|}{ Boys (n 139) } & \multicolumn{2}{|c|}{ Girls (n 150) } \\
\hline & Mean & SD & Mean & $\mathrm{SD}$ & Mean & SD \\
\hline Gestational age (weeks) & $38 \cdot 5$ & $3 \cdot 2$ & $38 \cdot 7$ & $3 \cdot 2$ & $38 \cdot 3$ & $3 \cdot 2$ \\
\hline Birth weight $(g)$ & 3051 & 494 & 3089 & 494 & 3016 & 494 \\
\hline Birth length $(\mathrm{cm})$ & $49 \cdot 2$ & $3 \cdot 3$ & $49 \cdot 4$ & $3 \cdot 6$ & $49 \cdot 0$ & $3 \cdot 0$ \\
\hline Head circumference (cm) & $33 \cdot 1$ & $2 \cdot 4$ & $33 \cdot 5$ & $2 \cdot 3$ & $32 \cdot 8$ & $1 \cdot 6$ \\
\hline Mother's age (years) & $24 \cdot 8$ & $6 \cdot 8$ & $24 \cdot 3$ & $6 \cdot 2$ & $25 \cdot 3$ & $7 \cdot 2$ \\
\hline$\%$ less than $2500 \mathrm{~g}$ at birth & \multicolumn{2}{|c|}{$11 \cdot 1$} & \multicolumn{2}{|c|}{$8 \cdot 6$} & \multicolumn{2}{|c|}{$13 \cdot 3$} \\
\hline$\%$ breast-fed & \multicolumn{2}{|c|}{$94 \cdot 7$} & \multicolumn{2}{|c|}{$98 \cdot 5$} & \multicolumn{2}{|c|}{$91 \cdot 2$} \\
\hline$\%$ solid food before 4 months & \multicolumn{2}{|c|}{$26 \cdot 6$} & \multicolumn{2}{|c|}{$25 \cdot 2$} & \multicolumn{2}{|c|}{$28 \cdot 0$} \\
\hline$\%$ solid food before 6 months & \multicolumn{2}{|c|}{$39 \cdot 5$} & \multicolumn{2}{|c|}{$34 \cdot 5$} & \multicolumn{2}{|c|}{$44 \cdot 0$} \\
\hline
\end{tabular}


compared the proportion of children being stunted (heightfor-age below $2 \mathrm{SD}$ of the reference median, $Z$ score $\leq-2$ ) and the proportion of children being underweight-for-age (weight-for-age below 2 sD of the reference median, $Z$ score $\leq-2$ ) according to the new WHO 2006, the CDC and NCHS growth standards/references. The $Z$ scores for the WHO 2006 standards were calculated using WHO Anthro 2005 software available on the WHO homepage (http:// www.who.int/childgrowth/software/en/). The $Z$ scores in comparison to the NCHS and the CDC growth references/ charts were calculated using the Epilnfo software package version $3 \cdot 2 \cdot 2$ (CDC, Atlanta, GA, USA).

\section{Results}

Our birth cohort consisted of 150 girls and 139 boys, briefly described in Table 1 . The mean birth weight for girls was $3016 \mathrm{~g}$ (range $1700-4170 \mathrm{~g}$ ), for boys $3089 \mathrm{~g}$ (range 1200-4510g). Thirty-two (11\%) children had a birth weight below $2500 \mathrm{~g}$ and one child had a birth weight below $1500 \mathrm{~g}$. Ninety-five per cent of the children were breast-fed but $27 \%$ of the mothers started to add solid food before the age of 4 months.

The proportion of children who were underweight-forage at 3,9 and 15 months is illustrated in Fig. 1. Using the CDC and the NCHS growth charts/references, a low proportion of children were underweight at 3 months $(0.7 \%$ and $1.0 \%$, respectively) but the proportion of underweight children seemed to grow considerably from 3 to 9 months and from 9 to 15 months of age (Fig. 1). When applying the new WHO 2006 growth standards, a higher proportion of children were underweight-for-age at 3 months but the proportion of underweight no longer seemed to accelerate with age. Figure 2 shows the mean underweight-for-age $Z$ scores obtained from age 3, 9 and 15 months using the WHO 2006, the CDC and the NCHS standards/references.

The proportion of children who were stunted at the age of 3, 9 and 15 months is shown in Fig. 3. The proportion of stunted children was $23.5 \%$ at 3 months, $22.5 \%$ at 9 months and $30 \cdot 5 \%$ at 15 months of age with the WHO 2006 standards, considerably higher than the proportions obtained with the CDC and the NCHS charts/references (Fig. 3). In line with the results on underweight-for-age, use of the NCHS references led to a pattern whereby the proportion of children being stunted increased from 3 to 9 months and from 9 to 15 months, a growth faltering pattern not observed when the WHO 2006 standards or the CDC charts were applied.

\section{Discussion}

Both the NCHS and the CDC growth references/charts had their data founded on relatively small sample sizes of infants, especially in the first six months of life, and an
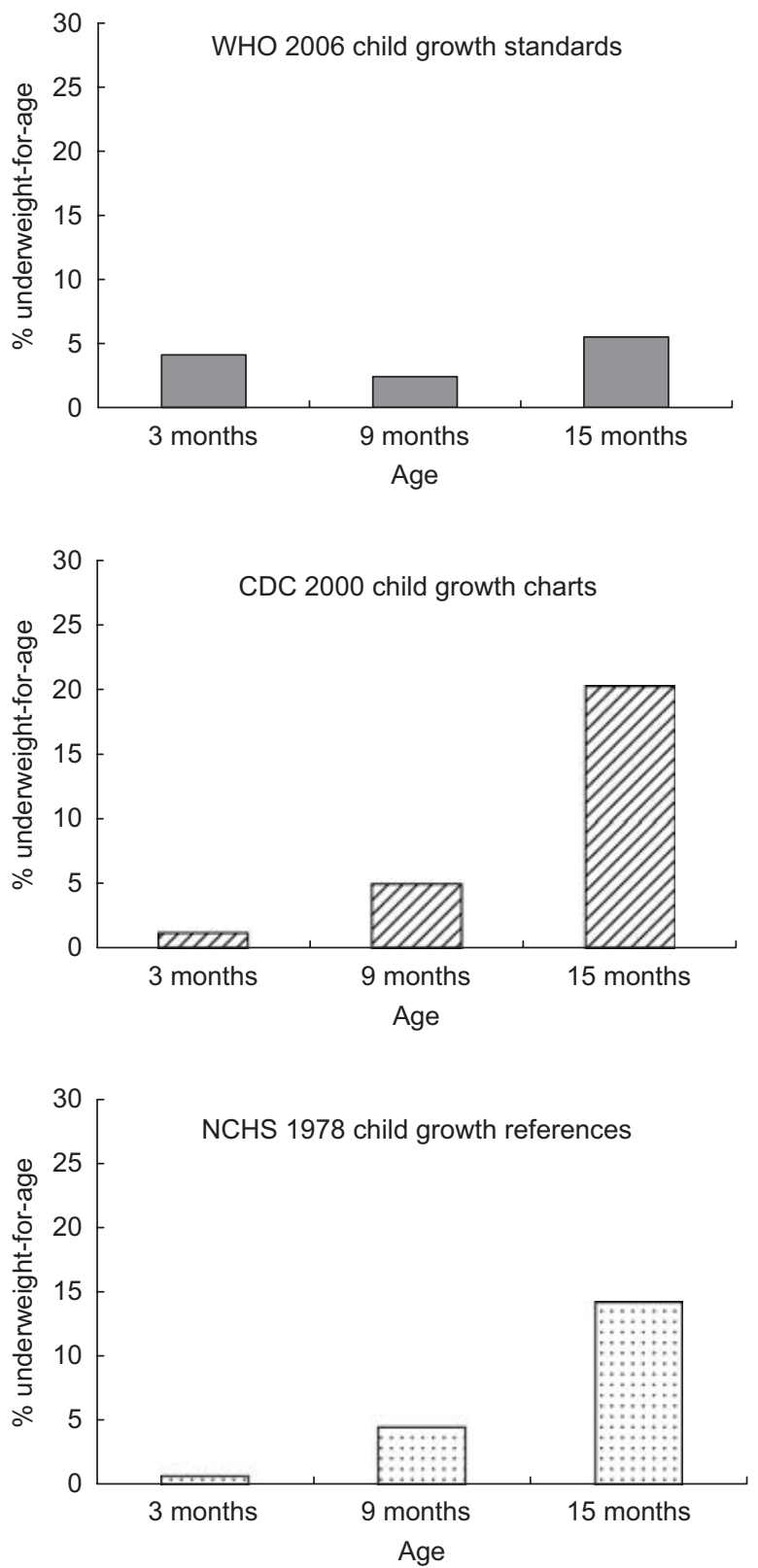

Fig. 1 Prevalence of underweight-for-age in 289 children from Lambaréné, Gabon, according to the WHO 2006 child growth standards, the Centers for Disease Control and Prevention (CDC) 2000 growth charts and the National Center for Health Statistics (NCHS) 1978 growth references

over-representation of formula-fed infants ${ }^{(2)}$. The WHO 2006 child growth standards are based on a much larger sample size of breast-fed infants from six different countries.

Use of the NCHS and the CDC child growth references led to a low proportion of malnourished children in early infancy that increased with age of the child. This growth faltering pattern has been described in numerous nutritional surveys all over the world ${ }^{(20)}$. The common interpretation was that children develop well while being 


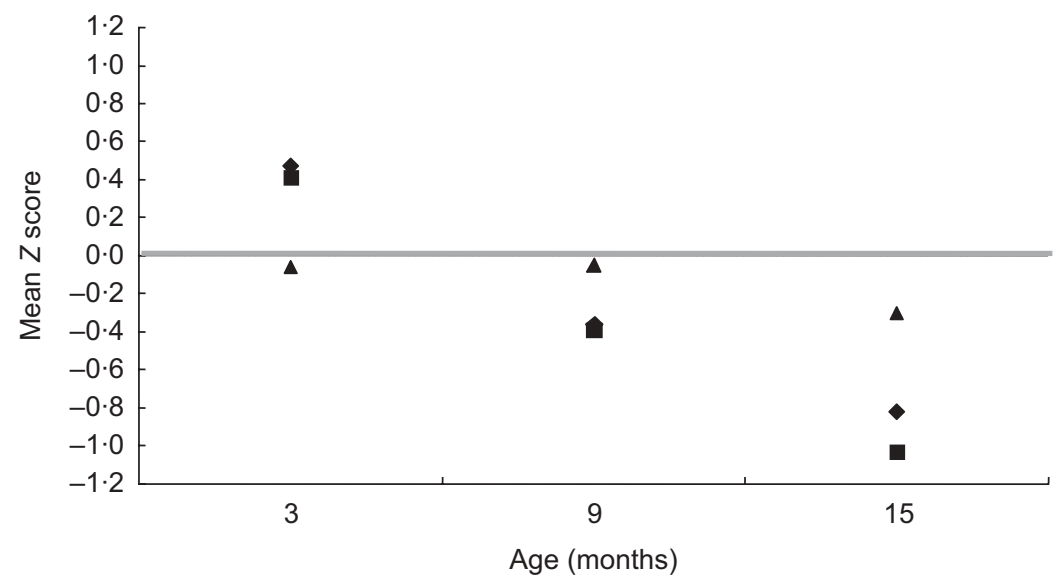

Fig. 2 Mean weight-for-age Z scores of 289 children from Lambaréné, Gabon, at 3, 9 and 15 months of age, using the WHO 2006 child growth standards $(\boldsymbol{\Delta})$, the Centers for Disease Control and Prevention (CDC) 2000 growth charts ( $\mathbf{\square})$ and the National Center for Health Statistics (NCHS) 1978 growth references $(\bullet)$

fully breast-fed in the first months of their life, but once additional feeding is necessary some of them start to become underweight. When applying the new WHO 2006 growth standards, a higher proportion of children were underweight-for-age at 3 months but the proportion of underweight children did not seem to accelerate with age $^{(20)}$, thus revealing that the classical growth faltering pattern of an increasing proportion of underweight children with age associated with the previously used reference charts was not observed once the WHO 2006 standards were applied.

We plotted the mean weight-for-age $Z$ score for the three measuring time points (3,9 and 15 months) of our birth cohort $v$, age in months (Fig. 2). With the NCHS and CDC references/charts, the 3-month-old children had the highest weight-for-age mean $Z$ score that subsequently declined considerably for the 9- and 15-month-old children. In contrast, the mean weightfor-age $Z$ score based on the WHO 2006 standards seemed to track around the same value. Although we had only three data points (3, 9 and 15 months) with spacing at 6 months, our graph is consistent with previous results where mean weight-for-age $Z$ scores were plotted $v$. age in a pooled sample of 226 healthy breast-fed infants from the USA and Northern Europe during their first year of life ${ }^{(3,4)}$. The lower variability around the line at $Z=0$ was consistent with the finding that the proportion of children being underweight-for-age varied less over time with the new WHO 2006 standards compared with the growth faltering pattern obtained with the NCHS or the CDC references/charts.

The WHO 2006 child growth reference charts are the first standards based on a data set from a multi-centre study specifically designed to generate child growth standards ${ }^{(6)}$. Nevertheless, the new WHO 2006 standards should not be adopted blindly, and concern has already been raised owing to the fact that the children in the six studies contributing data were highly selected for ideal growth conditions. They might therefore set height standards during the first two years of life and weight standards in the first six months that might not realistically be achieved by many children ${ }^{(21)}$. The prevalence of stunting and underweight-for-age in the present study was based on standardized anthropometric measurements conducted by trained health professionals. Reported prevalence of stunting and underweight-for-age in our sample of Gabonese infants and toddlers may therefore reflect the deviation from recommended feeding patterns ${ }^{(22)}$, i.e. early introduction of solid food, a common finding throughout most Central and West African countries ${ }^{(23-25)}$, and further unfavourable growth conditions. For the new WHO 2006 standards data from six different countries and from different ethnicities were pooled based on the assumption that child growth seems to depend much more on living conditions than on ethnicity $^{(26)}$. So far, however, the question remains whether there are ethnic/genetic differences in child growth ${ }^{(27)}$. Even small differences could become important when pooling large data sets and a pooled data set aimed to be representative for all children in the world might represent an artificial and fictional population, questioning the idea of one universal standard for the whole world.

The WHO 2006 growth standards are heavier and taller for the first six months of life than the NCHS or the CDC growth references/charts, explaining the high proportion of underweight and stunted children at the age of 3 months in our birth cohort when using the new WHO 2006 standards. In contrast, the proportion of 15-month-old children being underweight was lowest when using the new WHO 2006 standards. More than half of the children in this age group who had been classified as underweight with the NCHS or the CDC references were now classified to be within the normal weight range with the WHO 2006 standards. Either in the past too many 

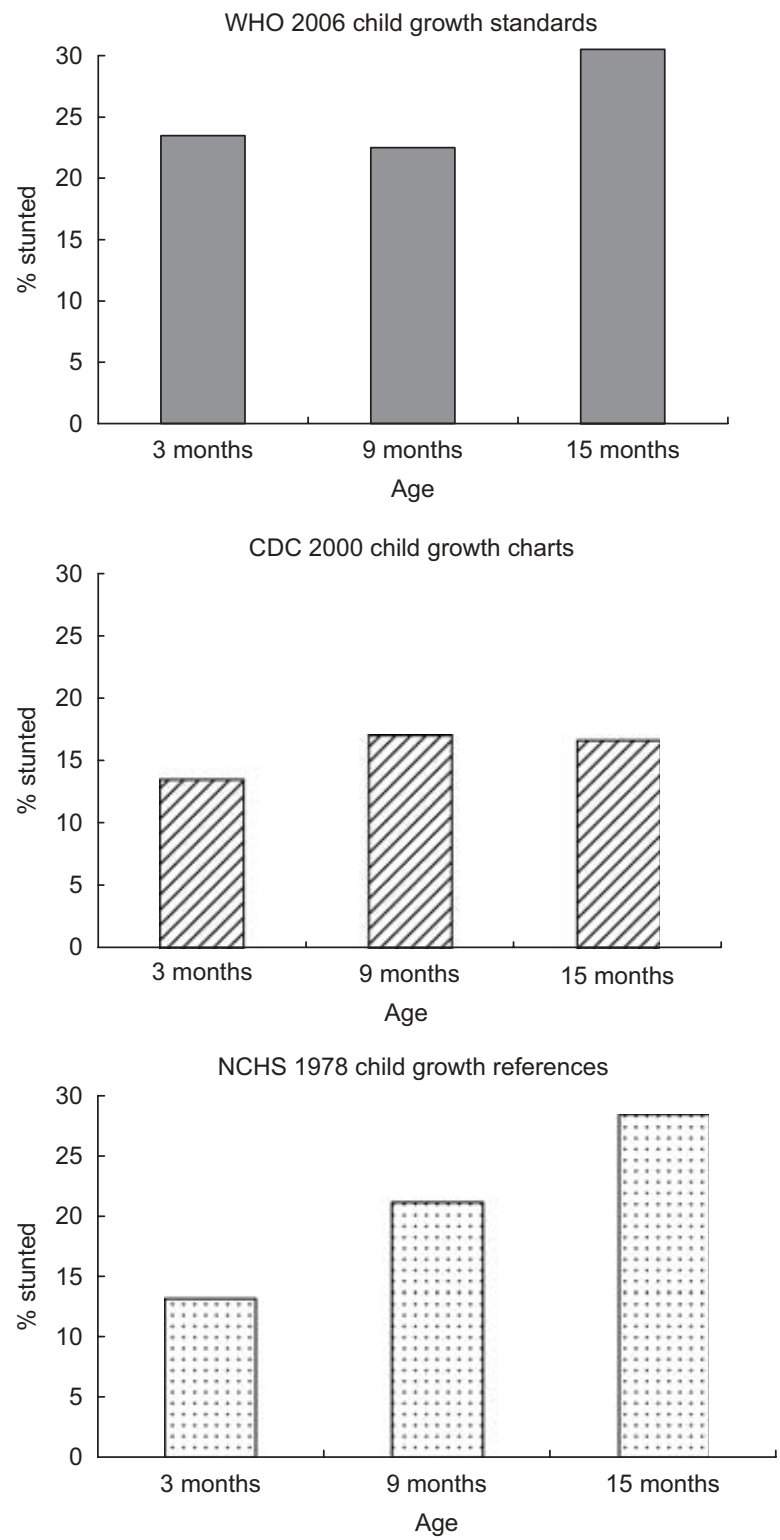

Fig. 3 Prevalence of stunting in 289 children from Lambaréné, Gabon, according to the WHO 2006 child growth standards, the Centers for Disease Control and Prevention (CDC) 2000 growth charts and the National Center for Health Statistics (NCHS) 1978 growth references

children above 1 year of age have been classified as underweight-for-age; or with the new WHO 2006 references some of the children who are underweight will be classified as being within the normal weight range, which could deny them a potentially favourable intervention.

Recently, first field-testing results looking at the concordance between clinical assessment (using visual classification codes for weight and height) and measurement-based assessment based on the WHO 2006 standards ( $Z$ score $<-2$ for underweight-for-age and stunting) were published ${ }^{(28)}$ followed by a comparative analysis applying the new WHO 2006 growth standards in populations in Bangladesh and the Dominican Republic, denoting results for underweight and stunting in line with the results presented in the current report ${ }^{(3)}$. Before abandoning the old NCHS reference charts that have been in use for nearly 20 years and thus profoundly shaped our knowledge about child growth ${ }^{(27)}$, we have to learn how to use the new WHO 2006 standards and what impact their use will have on nutritional assessment. Therefore, the introduction of the new WHO 2006 growth standards should be accompanied by comparisons with the old references, especially the NCHS reference. Those children falling into different categories depending on the standard being used will teach us about the benefits and pitfalls of the new standards.

\section{Conclusion}

The present results show different growth faltering patterns for the new WHO 2006 child growth standards compared with the NCHS and the CDC references/charts. With the new standards, much fewer children above the age of 1 year will be classified as underweight-for-age. On the other hand, among 3-month-old infants, the proportion being classified as underweight-for-age is much higher. Taking the new WHO 2006 standards leads to a higher proportion of stunted children in all age groups. Shifting to the WHO 2006 references may have considerable implications for child health programmes.

\section{Acknowledgements}

We thank all children and their parents/primary caregivers for participation in this study, and the Intermittent Presumptive Treatment in Infants study team for support. We gratefully acknowledge financial support from the Bill and Melinda Gates Foundation, the German Ministry of Education and Research (BMBF) and the German Academic Exchange Service (DAAD). There has been no conflict of interest for the work conducted.

Author contributions: N.G.S. analysed the study and wrote the first draft of the manuscript. K.K.G. designed the study, and K.K.G. and M.P.G. contributed to the analysis and the final version of the manuscript. All authors have significantly contributed to the conduct and day-to-day management of the study. B.L. and J.F. served as database managers. M.P.G. and P.G.K. designed the longitudinal study of which this nutritional survey is part.

\section{References}

1. Waterlow JC, Buzina R, Keller W, Lane JM, Nichaman MZ \& Tanner JM (1977) The presentation and use of height and weight data for comparing the nutritional status of groups of children under the age of 10 years. Bull World Health Organ 55, 489-498.

2. Kuczmarski RJ, Ogden CL, Guo SS, Grummer-Strawn LM, Flegal KM, Mei Z, Wei R, Curtin LR, Roche AF \& Johnson CL 
(2002) 2000 CDC Growth Charts for the United States: methods and development. Vital Health Stat 11 (246), $1-190$.

3. de Onis M, Onyango AW, Borghi E, Garza C \& Yang H (2006) Comparison of the World Health Organization (WHO) Child Growth Standards and the National Center for Health Statistics/WHO international growth reference: implications for child health programmes. Public Health Nutr 9, 942-947.

4. de Onis M, Garza C, Onyango AW \& Borghi E (2007) Comparison of the WHO child growth standards and the CDC 2000 growth charts. J Nutr 137, 144-148.

5. de Onis M, Garza C \& Victora CG (2003) The WHO Multicentre Growth Reference Study: strategy for developing a new international growth reference. Forum Nutr $\mathbf{5 6}$, 238-240.

6. de Onis M, Garza C, Victora CG, Onyango AW, Frongillo EA \& Martines J (2004) The WHO Multicentre Growth Reference Study: planning, study design, and methodology. Food Nutr Bull 25, S15-S26.

7. Borghi E, de Onis M, Garza C et al. (2006) Construction of the World Health Organization child growth standards: selection of methods for attained growth curves. Stat Med 25, 247-265.

8. Garza C \& De Onis M (1999) A new international growth reference for young children. Am J Clin Nutr 70, 169S-172S.

9. Dibley MJ, Goldsby JB, Staehling NW \& Trowbridge FL (1987) Development of normalized curves for the international growth reference: historical and technical considerations. Am J Clin Nutr 46, 736-748.

10. Graitcer PL \& Gentry EM (1981) Measuring children: one reference for all. Lancet 2, 297-299.

11. Waterlow JC (1979) Anthropometric assessment of malnutrition in children. Lancet 2, 250-251.

12. Dibley MJ, Staehling N, Nieburg P \& Trowbridge FL (1987) Interpretation of $Z$-score anthropometric indicators derived from the international growth reference. Am J Clin Nutr $\mathbf{4 6}$, 749-762.

13. Victora CG, Morris SS, Barros FC, de Onis M \& Yip R (1998) The NCHS reference and the growth of breast- and bottlefed infants. J Nutr 128, 1134-1138.

14. Hediger ML, Overpeck MD, Ruan WJ \& Troendle JF (2002) Early infant feeding and growth status of US-born infants and children aged 4-71 mo: analyses from the third National Health and Nutrition Examination Survey, 1988-1994. Am J Clin Nutr 72, 159-167.

15. de Onis M \& Onyango AW (2003) The Centers for Disease Control and Prevention 2000 growth charts and the growth of breastfed infants. Acta Paediatr 92, 413-419.
16. Grobusch MP, Lell B, Schwarz N et al. (2007) Intermittent preventive treatment in infants of malaria in Gabon: a randomized, double-blind, placebo-controlled trial. J Infect Dis 196, 1595-1602.

17. Klein Klouwenberg PM, Oyakhirome S, Schwarz NG et al. (2005) Malaria and asymptomatic parasitaemia in Gabonese infants under the age of 3 months. Acta Trop 95, 81-85.

18. Schwarz NG, Oyakhirome S, Potschke M et al. (2005) 5-day nonobserved artesunate monotherapy for treating uncomplicated Falciparum malaria in young Gabonese children. Am J Trop Med Hyg 73, 705-709.

19. Johnson TS, Engstrom JL \& Gelhar DK (1997) Intra- and interexaminer reliability of anthropometric measurements of term infants. J Pediatr Gastroenterol Nutr 24, 497-505.

20. Shrimpton R, Victora CG, de Onis M, Lima RC, Blossner M \& Clugston G (2001) Worldwide timing of growth falter ing: implications for nutritional interventions. Pediatrics 107, E75.

21. Binns C \& Lee M (2006) Will the new WHO growth references do more harm than good? Lancet 368, 1868-1869.

22. Kramer MS \& Kakuma R (2002) Optimal duration of exclusive breastfeeding. Cochrane Database Syst Rev (1), CD003517.

23. Lauer JA, Betran AP, Victora CG, de Onis M \& Barros AJ (2004) Breastfeeding patterns and exposure to suboptimal breastfeeding among children in developing countries: review and analysis of nationally representative surveys. BMC Med 2, 26.

24. Bloss E, Wainaina F \& Bailey RC (2005) Prevalence and predictors of underweight, stunting, and wasting among children aged 5 and under in western Kenya. J Trop Pediatr 50, 260-270.

25. Mamabolo RL, Alberts M, Mbenyane GX, Steyn NP, Nthangeni NG, Delemarre-Van De Waal HA \& Levitt NS (2004) Feeding practices and growth of infants from birth to 12 months in the central region of the Limpopo Province of South Africa. Nutrition 20, 327-333.

26. Bhandari N, Bahl R, Taneja S, de Onis M \& Bhan MK (2002) Growth performance of affluent Indian children is similar to that in developed countries. Bull World Health Organ 80, 189-195.

27. Waterlow J (2006) Difference between the 'old' NCHS reference as 'descriptive' and the new WHO reference as 'prospective'. Public Health Nutr 9, 662.

28. Onyango AW, de Onis M, Caroli M, Shah U, Sguassero Y, Redondo N \& Carroli B (2007) Field-testing the WHO child growth standards in four countries. J Nutr 137, 149-152. 